\title{
Du rite, de sa production et de ses usages : pratiques et spéculations rituelles en Chine ancienne
}

\section{Benoît Vermander}

\section{(2) OpenEdition Journals}

Édition électronique

URL : https://journals.openedition.org/assr/27233

DOI : $10.4000 /$ assr.27233

ISSN : $1777-5825$

Éditeur

Éditions de l'EHESS

Édition imprimée

Date de publication : 1 octobre 2015

Pagination : 147-178

ISBN : 978-2-7132-2515-4

ISSN : 0335-5985

Référence électronique

Benoît Vermander, « Du rite, de sa production et de ses usages : pratiques et spéculations rituelles en Chine ancienne », Archives de sciences sociales des religions [En ligne], 172 | octobre-décembre, mis en ligne le 26 avril 2018, consulté le 15 février 2023. URL : http://journals.openedition.org/assr/27233 DOl : https://doi.org/10.4000/assr.27233 


\title{
Benoît Vermander
}

\section{Du rite, de sa production et de ses usages: pratiques et spéculations rituelles en Chine ancienne}

\author{
À propos de : \\ Bolleau Gilles, Politique et rituel dans la Chine ancienne, Paris, Collège \\ de France, Institut des Hautes Études Chinoises, Bibliothèque de \\ l'Institut des Hautes Études Chinoises, vol. XXXVII, préface de \\ John Lagerwey, postface de Roberte Hamayon, 2013, 481 p. \\ Et références complémentaires à : \\ BRIndley Erica Fox, Music, Cosmology, and the Politics of Harmony \\ in Early China, Albany, SUNY Press, 2012, 237 p. \\ Hong Tao, Xin shu yu zhi dao (L'art du coeur et la voie du gouverne- \\ ment), Shanghai, Presses populaires de Shanghai, 2013, 195 p. \\ ING Michael David Kaulana, The Dysfunction of Ritual in Early \\ Confucianism, Oxford, Oxford University Press, "Oxford Ritual \\ Studies Series », 2012, 304 p. \\ VANDERMEersch Léon, Les deux raisons de la pensée chinoise, Divi- \\ nation et idéographie, Paris, Gallimard, coll. "Bibliothèque des \\ Sciences humaines », 2013, 208 p. +16 p. h.t.
}

La présente note porte sur la production et les fonctions de l'activité rituelle en Chine antique comme sur la façon dont cette activité a été comprise et représentée dans cette même civilisation. La lecture d'un ouvrage récent, celui de Gilles Boileau, menée de concert avec celle de quelques autres publications, peut contribuer à insérer les recherches sur le système politico-religieux de la Chine ancienne dans le domaine des études rituelles. Qu'il soit bien spécifié dès le départ que cette note ne se fonde pas sur une recension complète des études poursuivies dans le domaine géographique et historique précité. Les ouvrages choisis l'ont été simplement pour apporter des éclairages spécifiques. Il s'agit donc bien d'une "note critique ", en aucun cas d'un état des travaux récents dans un champ qui reste en état de renouvellement permanent. 


\section{Le rituel, point nodal d'un complexe politico-religieux}

L'affirmation posée par Gilles Boileau à l'ouverture de son ouvrage en définit d'emblée l'objet et la portée : "Le rite est l'expression primordiale du déploiement historique concret des institutions sociales, politiques et religieuses de la Chine anciennes. » (p. 11) Elle permet aussi d'entrevoir pourquoi sa tentative est susceptible d'intéresser des publics différenciés : à un premier niveau, elle propose une synthèse d'anthropologie historique de la Chine ancienne dont l'ambition pourra rappeler celle d'un Granet ; l'ampleur de la documentation et le traitement exemplaire des sources légitiment amplement le projet, et l'ouvrage de Gilles Boileau est à lire d'abord comme un essai majeur sur le système politicoreligieux de la Chine antique. À un deuxième niveau, l'ouvrage est en dialogue constant avec des travaux d'anthropologie historique poursuivis en d'autres contextes, et s'inscrit donc dans une perspective comparatiste qui amène à en recommander la lecture à des spécialistes d'autres champs géographiques et historiques, cela d'autant plus que l'érudition déployée n'entache pas la clarté de la langue ni la modestie dans la formulation des aperçus ouverts. Enfin, ce deuxième niveau de lecture peut aisément se prolonger dans une réflexion ouverte, plus spéculative, sur la nature et les fonctions du rituel comme sur les articulations du politico-religieux. Une fois exposées les principales thèses de l'ouvrage, c'est dans pareille réflexion que j'essayerai de m'engager. Ce faisant, je me réfèrerai donc à quelques publications dont la lecture est susceptible de compléter ou questionner la synthèse proposée par l'A.

Il s'agit bien ici de retracer " un déploiement historique concret ", et le plan de l'ouvrage est largement chronologique. Les premier et deuxième chapitres sont consacrés à la figure du $w u$ (chamane, devin, guérisseur) et du roi, respectivement, à l'articulation de la Chine des Shang (XVII ${ }^{\mathrm{e}}-\mathrm{XI}^{\mathrm{e}}$ av. J.-C.) et des Zhou (1046 av. J.-C - 256 av. J.-C., encore que la dynastie n'exerce plus de souveraineté effective après 771). Les deux chapitres suivants étudient de près les textes relatifs aux sacrifices et aux offrandes, reliant les enseignements qu'ils contiennent au fonctionnement et aux dysfonctionnements de la dynastie Zhou. C'est dans le contexte des troubles qui marquent la période des Printemps et Automnes (771-481 av. J.-C.) qu'apparaît la figure des shi (petits nobles, Lettrés), lesquels, regroupés en écoles concurrentes penseront à frais nouveaux le système rituel des royaumes auxquels ils font allégeance (chapitre 5). L'évolution se poursuivra jusque dans la nouvelle synthèse rituelle et politique offerte par l'Empire Qin, presque immédiatement suivie et corrigée par celle présidant à l'établissement de la dynastie Han (chapitre 6). Ne pouvant suivre ici l'ouvrage en tous ses développements, résumons ses thèses les plus saillantes :

1) Dès la dynastie Shang, la hiérarchie sociale est dominée par la personne du roi (wang). La figure du $w u$ est à penser en relation systémique avec celle du souverain. Les $w u$ médiatisent et réalisent l'alliance conclue par le souverain avec les divinités naturelles du royaume (montagnes, fleuves) au travers de modalités 
diverses, lesquelles peuvent inclure le sacrifice de femmes $w u$. Cette politique d'alliances ritualisées vise à éviter ou juguler les catastrophes qui peuvent affecter les territoires largement non encore domestiqués sur lesquels on exerce prédation. Si le sexe des $w u$ dont parlent les textes n'est pas toujours identifiable, les $w u$ masculins sont d'abord associés aux activités guerrières, tandis que les danses des $w u$ féminines ont à voir avec la délicate gestion des phénomènes météorologiques extrêmes (sécheresses et inondations). (J'ai résumé un article antérieur de l'A., des publications apparentées, et le débat sur le rapprochement entre $w u$ et chamane dans : "Chamanismes de l'Asie de l'Est. Termes et rôles en mouvement ", Assr, 160, octobre-décembre 2012 - voir notamment p. 36-41.) La postface de Roberte Hamayon reprend et commente la comparaison/distinction ainsi dressée entre les sociétés de la Chine antique et celles du monde sibérien.

2) Ces alliances ritualisées ne doivent pas être représentées sur un modèle directement matrimonial : les femmes offertes sont "marquées » du côté de la nature (comme le sont les êtres difformes qu'on leur substitue parfois), tandis que le roi joue plutôt un rôle de moyen terme entre nature et culture, susceptible comme tel de se déployer en des fonctions diverses et au travers de figures complémentaires ( $w u$, ministres). À cet égard, l'affaiblissement de la royauté sous les Printemps et Automnes va de pair avec les remaniements qui interviennent dans les rapports idéels et matériels entre nature et société, même si le rapport instauré entre le corps du souverain d'une part, le corps du territoire dont montagnes et fleuves constituent l'ossature d'autre part, se perpétuera sous l'Empire.

3) En parallèle, les attentes dont «le corps du souverain » fait l'objet établissent ce dernier en point nodal: la pratique sacrificielle que sa singularité régule fait passer de la multiplicité de la nature à l'unité organisée et hiérarchisée du social. Développé tout au long de la dynastie des Zhou, le modèle sacrificiel voit dans le rituel le «pédagogue de la civilisation » (p. 15) par lequel l'humanité fait l'anamnèse de sa sortie du règne animal et de son avènement. La découpe sacrificielle est l'image d'une opération mentale analogue.

4) La rupture symbolisée par la découpe se trouve inversée au travers de l'ingestion de parties animales par tous les participants, opération destinée à symboliser et réaliser la cohésion du groupe, dans un modèle qui associe exigence d'unanimité et principes hiérarchiques. En même temps, la viande des victimes dont le partage assure la cohésion est représentée comme richesse octroyée par les ancêtres. L'entame des viandes est toujours effectuée par les ancêtres ou leurs représentants, tandis que les autres participants n'étaient censés qu'en goûter, jusqu'à transmission totale des restes au niveau le plus bas. Cette chaîne descendante faisait du roi, maître et distributeur des richesses, celui qui goûtait le plus modérément aux dons provenant des ancêtres. Le sacrifice était donc image inversée de l'ordre social effectif. La supériorité sociale était étroitement associée à la capacité de donner. 
5) Cette capacité à donner est redoublée par le pouvoir de conserver pour donner plus tard : le pouvoir le plus grand est celui de différer le don. La crise politique affectant les Zhou au IX ${ }^{\mathrm{e}}$ siècle est liée aux critiques adressées à la maison royale : celle de ne pas accorder à ses soutiens nobles les moyens de leur continuité. Les anomalies rituelles relevées par les textes fonctionnent alors comme informations historiques d'importance particulière. La crise rituelle est toujours symptôme de crise politique.

6) Les transformations affectant dès lors par vagues successives les régulations rituelles se sont accompagnées de la montée en puissance de la plus basse classe de la noblesse $(s h i)$. Spécialistes rituels employés par des états rivaux, les shi ont transformé leur savoir (ou "intelligence»- je discute plus bas l'usage de ce terme par l'A.) en capital politique. L'effervescence intellectuelle résultant du processus de compétition ainsi enclenché est porteuse de pluralisation mais aussi, paradoxalement, de capacité accrue à l'hégémonie en tant qu'elle aura permis aux États de penser leur propre pouvoir. La maîtrise du discours, une fois qu'elle est usurpée par le souverain, fait de ce dernier la source de tout magistère. Si, peu avant l'apparition du Premier Empire, la nostalgie des rituels anciens refait surface, elle est vite submergée par des exigences de normalisation, d'impartialité, de rationalisation qui modifient la matrice théologico-politique de la Chine ancienne. Dans cette période transformative, la fonction souveraine est pensée comme double : réactivité face à l'urgence, et garant de stabilité. Pour assurer cette dernière exigence, les rites sont vus comme essentiels parce qu'ils transmettent des normes supposées émaner du plus lointain passé, et, comme telles, indiscutables. La part " réactive » ou simplement " active » du pouvoir est largement dévolue au ministre principal, tandis que la stabilité procurée par le souverain est assimilée à son insertion dans la continuité même du cosmos. On voit ici l'écart peu à peu creusé avec l'ancien modèle de générosité et unanimité organiques. Le fondateur de la dynastie des Han tirera la leçon des évolutions intervenues en ritualisant son pouvoir de façon telle à assurer la continuité de l'Empire.

7) Dans sa conclusion, l'A. s'essaie à penser la pérennité de l'importance accordée au rituel en Chine: pour les affaires de gouvernement, rien de plus pressant que le rite, déclare le Livre des Rites (Liji, chapitres Jitong et Liyun). Le Shuowen jiezi (dictionnaire chinois achevé au tout début du deuxième siècle de notre ère) définit le rite (li) par sa mise en œuvre (lü) (cit. p. 421-422). (Ajoutons ici deux précisions à l'analyse de l'A. : lü se prononce li à l'époque, et la force de l'identification est donc redoublée par le fait d'établir un homonyme en synonyme ; l'hexagramme désigné par ce terme s'applique à un moment périlleux dans lequel on conjure les dangers.) La réflexion sur le rite poursuivie dans la Chine ancienne voit donc en lui un savoir littéralement "incorporé » qui introduit du fixe et de l'ordre dans l'inconstant et dans le fluide tout en opérant une transformation de celui qu'on agrège à l'opération rituelle. (C'est à cet endroit 
seulement - p. 426-427 de l'ouvrage - que l'A. pose la question de «l'intentionnalité religieuse » du rite. Nous reviendrons sur ce point plus avant.) Si le pouvoir était en mesure d'instrumentaliser le rite - et ne se privait pas de le faire -, les auteurs chinois l'avaient en même temps théorisé comme exerçant une fonction régulatrice de contrôle, d'apaisement et de limitation des appétits. Gilles Boileau cite cette phrase en tout point remarquable: "Quelqu'un pourrait-il tenir quelque chose de chaud sans avoir auparavant humecté ses mains? Le rite est au gouvernement ce que l'humidité est à la chaleur. "(Zuozhuan, la première partie de la phrase étant une reprise l'Ode Sangrou, cit. p. 430-431.) Le rite était bien en mesure de tenir pareil rôle, conclut l'A., d'une part parce qu'il orientait l'homme vers ses origines, d'autre part parce que la ritualisation religieuse du politique pouvait aussi être utilisée contre l'arbitraire de l'État.

Une « mise en thèses » s'apparente à une mise en bière. En d'autres termes, la lecture de l'ouvrage opérée à l'instant ne saurait lui faire justice : le plaisir de la lecture consiste souvent à partager celui qu'éprouve l'auteur à scruter ses sources. Ces dernières évoluent avec les époques parcourues. Au début du livre, l'attention porte sur les inscriptions oraculaires comme sur les travaux des universitaires chinois qui se sont spécialisés dans leur interprétation. Des compilations dont la rédaction s'étale souvent sur plusieurs textes complètent ce matériau de base - notamment le Guоyu ( "Discours des royaumes ", fin du cinquième siècle av. J.-C.), le Lüshi chunqiu ("Annales des Printemps et des Automnes de Lü ", compilé au début des Qin), le Zuozhuan (important commentaire des "Annales des Printemps et des Automnes de Lü») et bien entendu le Zhouli ( "Rites des Zhou »), le Yili ( Rites cérémoniels») et surtout le Liji ( Mémoire sur les rites ", ouvrage dont la datation fait problème mais dont certaines parties sont fort anciennes). La lecture effectuée excelle à nouer ensemble lieux, espèces biologiques ou personnages évoqués, coutumes décrites et mythes récurrents pour évoquer la conquête rituelle et symbolique d'un territoire naturel qui, en Chine archaïque, échappait encore largement au contrôle humain. L'A. manie ses textes avec prudence, et sait pertinemment qu'ils parlent souvent de l'époque de leur rédaction ou compilation sous couvert de rapporter les faits et rites du passé, mais il sait aussi y trouver des indices probants, déposés par une longue sédimentation textuelle (excellentes remarques sur l'appréciation des sources p. 151-153). Dans l'ensemble du corpus, l'évolution de la place faite aux $w u$ et leurs rituels parle de la frontière mouvante entre civilisation et chaos, ordre social et désordre naturel, la gestion de la mort et des funérailles constituant en quelque sorte l'extrême frontière. C'est sur cette même frontière que se situait le roi, mais il y était placé pour assurer la continuité et la subsistance du corps social vivant et ordonné, tandis que les $w u$ contribuaient à cette même survie de par leur relation ambiguë, complice avec les forces de mort qui la menacent.

Les deux chapitres les plus « savoureux » sont, comme il se doit, ceux consacrés à la cuisine du sacrifice. Son étude attentive s'impose, puisque le Liji nous 
rappelle explicitement qu' " au commencement du rituel il y a d'abord la boisson et la nourriture» (chapitre Liyun, cit. p. 149). L'analyse des offrandes, des séquences et des plats servis, et notamment de ce qui est offert respectivement aux ancêtres et aux participants, montrent bien le caractère de mémorial joué par le rituel, le passé le plus lointain rapproché du «naturel » (quelle que soit l'appréciation - variable - portée sur la période précédant l'instauration de la "culture »). L’importance accordée aux opérations de différenciation (différenciation et humanisation constituant un processus conjoint) est ainsi illustrée par le paragraphe suivant du Liji: "Le bœuf destiné à la divinité suprême est isolé dans un enclos pendant trois mois. Le bœuf destiné au dieu du millet n'a pas besoin d'être isolé. Ceci afin de différencier le culte aux esprits célestes de celui des ancêtres. » (Chapitre Jiaotesheng, cit. p. 175) L'ordre instauré reflète celui même du cosmos, en même temps qu'il en permet la perpétuation. Les « cuisines du sacrifice » en monde chinois et grec diffèrent par la façon dont elles s'inscrivent dans des modèles contrastés de délibération et différenciation politiques, mais présentent des similitudes quant aux processus historiques qui furent à l'origine des réformes rituelles (p. 220-225). Par ailleurs, en Chine l'apparition de la classe des Lettrés réinterprète le processus de don à origine sacrificielle dans un processus d'échange au travers duquel l'intelligence est récompensée et " nourrie ».

Au cours des chapitres 3 à 6, l'A. ajoute à ses sources celles émanant des Classiques élaborés justement autour du milieu shi, principalement les Analectes, le Mencius, le Xunzi, et le Zhuangzi. Mais un indice peut amener à questionner certains aspects de son entreprise : c'est moins la synthèse intellectuelle offerte par ces textes quant à la nature et aux fonctions du rituel qui est ici retracée que la façon dont les mêmes textes nous alertent sur les transformations historiques à l'œuvre. Type de lecture légitime, qu'on pourra qualifier d'historico-critique, mais ce choix méthodologique de l'A. va guider les questions que j'entends désormais adresser à l'ouvrage. Il s'agit moins, on s'en doute, de vouloir réduire la portée d'un ouvrage remarquable que de rechercher comment compléter et prolonger l'approche d'anthropologie historique qu'il développe.

\section{Musique et rituel}

Une précision s'impose : les travaux de Gilles Boileau n'empruntent en rien aux ritual studies. On chercherait en vain une référence aux ouvrages de Catherine Bell ou de Stanley Tambiah par exemple (la première pourtant spécialiste des religions chinoises). De ce fait peut-être, les définitions générales du rite sont plus pauvres que l'analyse empirique développée dans l'ouvrage ou même que ce suggèrent les aperçus des penseurs chinois qui y sont cités. Dans sa conclusion, Gilles Boileau décrit le rituel comme "une série de gestes, de paroles et d'actes formalisés, dont la mise en œuvre définit un ordre à la fois spatial, temporel et social » (p. 421). Il l'insère dans la continuité de « l'habitude individuelle » 
et des «normes sociales " comme une " mise en scène explicite des corps, dans un temps et un espace qu'il définit et oriente » (p. 423). Approche poursuivie un peu après par « une définition du rite comme norme culturelle et corporelle des interactions de l'humain avec le monde extrahumain » (p. 427). Ces définitions conclusives ne semblent pas faire entièrement justice au contenu du matériau et des analyses. La dimension corporelle du rite est de fait devenue un des leitmotiv des études rituelles, mais elle s'accompagne d'une insistance sur ce que fait le rite par son inscription corporelle : il ne se contente pas de manifester et d'internaliser un ordre social et cosmique donné, il le construit, le solidifie ou bien le transforme. Loin de seulement normer, il opère. La performance rituelle, par son travail sur les actes et les symboles qu'elle met en œuvre, ouvre à des "sens émergents » par lesquels la communauté redéfinit ses frontières, son fondement et sa destinée.

De ce point de vue, le Shuowen jiezi qui définit le rite par sa mise en œuvre même dit sans doute davantage encore que ce que l'A. y a trouvé. Le suivi des témoignages textuels qu'effectue ce dernier est si précis qu'on se demande si une «mise à distance » ne s'imposait pas par endroits, justement pour évaluer autrement leur richesse. Or l'A. manifeste en même temps une certaine méfiance par rapport aux realia, en tout cas par rapport à l'interprétation par les archéologues eux-mêmes des témoignages exhumés. Sa position est finement argumentée (p. 179-189) et introduit à un débat relatif à des problèmes de datation qui l'oppose au spécialiste de la musique rituelle chinoise archaïque, Lothar Von Falkenhausen (p. 192-198). Ce n'est pas ce débat lui-même qui nous intéresse ici, mais bien plutôt ce qu'il signale : creusée par la distance prise avec l'archéologie, la discrétion gardée sur le rapport entre performances musicale et rituelle, et ce malgré leur liaison intime dans tous les textes utilisés. À titre d'exemple, le parallélisme des entrées en matière des chapitres "Sur les rites » et "Sur la musique » du Xunzi - lesquels chapitres se suivent et forment un tout - est frappant : (a) les hommes ne peuvent pas ne pas être sujets à des désirs violents et le rite est là pour en réguler l'expression et les conséquences ; (b) les hommes ne peuvent pas ne pas éprouver de sentiments puissants, et la performance musicale est là pour en réguler l'expression et les conséquences. Certes Gilles Boileau cite, entre quelques autres, la phrase clé du Liji selon laquelle « la musique unifie, le rite distingue ", phrase qui précise aussi que l'unité crée familiarité (qin) mutuelle et la distinction respect (jing) mutuel (chapitre Yueji, cit. p. 177), mais les références à la performance musicale ne vont guère plus loin et n'inspirent pas de développement indépendant.

Quittons ici provisoirement son ouvrage pour résumer l'approche qui est celle d'Erica Brindley dans la publication signalée en bibliographie : Brindley voit dans les textes antérieurs à 325 av. J.-C. environ une conception de l'harmonie (he) fondée avant tout sur des pratiques culturelles - musicales, culinaires, rituelles -, conception qui régule la rhétorique et les pratiques politiques. L'excellence et le goût déployés dans la performance musicale sont indice du degré de 
stabilité et de pérennité qu'un État peut espérer atteindre (plusieurs passages du Zuozhuan notamment corroborent cette analyse). C'est vers le temps de la rédaction du Zhuangzi que le terme Harmonie s'étend à l'édifice cosmique, la "flûte du ciel " (tian lai) dominant la musique émise par les flûtes de la terre et des hommes. La Voie du cosmos est musique, et la Voie de la musique est cosmique (appuis textuels dans divers textes du Lüshi chunqiu, du Mozi, et du Huainanzi). Aussi est-il de grande importance pour notre sujet que le chapitre Yueji du Liji fasse de l'échange des énergies ( $q i$ - un concept formalisé tardivement et dont la formulation autorise les spéculations cosmologiques sur la musique) entre la Terre et le Ciel un acte indissociablement musical et rituel. La musique étant le « moteur " du cosmos, la performance musicale rituelle contribue au bon fonctionnement de ce dernier. Si l'on accepte le schéma temporel défendu par Bradley (lequel me semble concorder avec ce qu'on sait par ailleurs), la conséquence est d'importance : loin de voir son importance symbolique diminuer avec le temps, la performance rituelle et sa dimension musicale reçoivent dans la pensée chinoise une place de plus en plus centrale. Peut-être faut-il préciser que ce que la performance rituelle perd en importance politique relative (au profit des « châtiments » $f a$, dont l'échelle, annoncée publiquement, n'est plus du ressort du caprice princier), elle le gagne en centralité cosmique. Contrairement à une hypothèse qui verrait le rite devenir un simple moyen politique au fur et à mesure de son évolution, on pourrait alors parler d'une "dépolitisation » relative du rituel et de son inscription progressive dans un ordre spécifiquement cosmique et religieux. La compréhension évolutive de la performance musicale serait un indice probant de cet état de fait. À cet égard, il est frappant que la description de la création de la musique rituelle par l'Empereur Jaune dont la rhétorique soit la plus solennelle et la plus «cosmique » se trouve dans un texte du légiste Hanfeizi (chapitre Shiguo). Mais il faut rester prudent dans l'interprétation des faits : on peut aussi suggérer que la dimension cosmique conférée progressivement à la performance musicale ait à voir avec l'idée que le domaine d'action du gouvernant n'était pas enfermé dans les étroites limites de l'Empire mais s'étendait à tout ce qui se trouve sous le Ciel (tian xia), une évolution qui annoncerait l'avènement de l'Empire Qin. Les deux hypothèses ne sont pas contradictoires : la majesté cosmique et impériale accrue du rituel irait de pair avec la réduction de son effectivité politique (mais non point forcément avec une diminution de son rôle comme éducateur de l'intériorité).

Mentionnons certains textes relevés par Erica Brindley, lesquels vont à l'appui du schéma proposé : dans le "Canon de l'Empereur Shun » du Livre des Documents (Shujing) le Maître de musique royal est représenté comme celui qui codifie (dian) la relation entre humains et esprits par la codification musicale même. Sur cette base, la performance musicale est vue comme permettant de "reposer dans la vertu " (an de), le respect de la codification définissant la qualité de la gouvernance (cf. p. 27-32). Dans le Lüshi chunqiu, la rhétorique 
employée évolue notablement : elle suggère que la performance musicale peut et doit jouer sur la psyché du gouvernant de telle façon à aligner cette dernière sur le mouvement cosmique du Dao (un style musical débridé provoquant, comme on peut l'imaginer, l'effet inverse). Le Guoyu décrit la musique comme la force qui libère les vents sur les montagnes en même temps qu'elle étend et exalte le champ d'action de la vertu (cf. cit. p. 51). Bien entendu, dans les Analectes, la performance musicale était déjà présentée comme un indispensable medium pour la perfection de l'esprit et de la conduite. Mais par la suite, une idée complémentaire prolongera et infléchira cette approche : la musique est ce qui permet au sage d'entrer en résonance, dans le microcosme de son corps, avec l'harmonie du cosmos entier. Culture morale et participation à l'harmonie cosmique s'associent par le biais de l'apprentissage musical (p. 125-9).

L'attention au lien qui unit musique et rituel doit nous mener plus loin encore : Fingarette avait noté avec raison que, chez Confucius, le rapport entre li (rituel) et ren (vertu d'humanité) est conçu sur le modèle de la performance musicale : la réussite d'un rituel comme la performance d'un acte d'humanité sont analogues à celles d'un musicien, dans laquelle on pourra percevoir comme d'instinct confiance et sincérité (ou leur absence). (Herbert Fingarette, Confucius: The Secular as Sacred, New York, Harper Torchbooks, 1972, p. 53-54.) C'est sur ce fond d'une attention à la justesse de la performance qu'on peut interpréter une phrase telle que : "Si un homme est dépourvu d'humanité, que lui servent les rites? Si un homme est dépourvu d'humanité, que lui sert la musique ?» (Analectes, 3, 3) Notre excursus sur la relation symbiotique entre musique et rituel nous ramène ainsi vers le livre de Gilles Boileau, ce pour lui adresser la question suivante : dans le schéma proposé, que faire de la dimension spécifiquement spirituelle et religieuse du rituel ?

\section{L'intentionnalité du rite}

La pertinence du terme "spécifiquement » utilisé à l'instant peut être questionnée. Gilles Boileau écrit : «La question de savoir si, dans la Chine ancienne, l'attitude spirituelle précédait la pratique ou si elle en procédait ne peut être posée qu'à condition d'ignorer la nature des moyens spécifiques aux rites. Ils comprenaient l'ensemble des éléments sensuels [...] par le truchement desquels le rite déployait sa puissance ; ces moyens créaient les conditions de l'expérience religieuse sans que la relation entre moyens et fin soit univoque. » (p. 428) On peut noter que cette phrase introduit moins de «circularité » dans l'analyse qu'elle l'affirme : clairement, pour l'A. l'attitude spirituelle est davantage un produit de la performance rituelle que son préalable. En tout état de cause, et même en tenant compte de la justesse intrinsèque de la remarque, la question de la nature de l'expérience que la performance rituelle était supposée produire ou accompagner n'est pas abolie, et se pose peut-être même avec une insistance accrue. 
On a déjà pressenti que les textes anciens eux-mêmes développent une réflexion approfondie sur le sujet, y compris sur l'alliance entre sentiments de distinction/ séparation provoqués par la gestique rituelle et sacrificielle et sentiment d'union produit par la performance musicale qui l'accompagne.

J'ai déjà noté que l'une des phrases les plus célèbres des Analectes ne faisait son apparition dans l'ouvrage qu'à la page 426. (Au passage, l'absence d'une table récapitulative des citations est dommageable ; et l'index général ne compense pas cette absence : si le Daodejing par exemple y est référé, ni les Analectes ni le Liji ne le sont.) Citons ce passage en sa totalité : « [le Maître] sacrifiait [aux ancêtres] comme s'ils étaient là ; il sacrifiait aux esprits comme s'ils étaient là. Il disait : "Si je ne suis pas présent au sacrifice, c'est comme si je ne sacrifiais pas.” " (Analectes 3, 11) L'A. voit dans l'attitude intérieure recommandée par Confucius une ouverture vers une compréhension "culturelle " de la sphère cultuelle ; ouverture qui n'aurait guère été suivie d'effets dans la suite de l'histoire chinoise. Il me semble que c'est là mésestimer tant l'impact que la portée théorique du passage. Il faut savoir le prendre comme un tout plutôt que d'opérer comme le font nombre de commentateurs, dont l'A.: selon eux, le premier membre de phrase porterait sur l'attitude intérieure de Confucius, qui déciderait de prêter hommage aux esprits quoi qu'il en soit de leur existence; le second nous rappellerait qu'il désapprouvait le fait que certains nobles s'étaient mis à déléguer la présidence du sacrifice à des intermédiaires et s'abstenaient d'y être présents. Or, à mon sens, le point essentiel du passage réside en ce que la présence du participant et celle des esprits se répondent l'une à l'autre, s'appellent l'une l'autre. La présence n'est pas de l'ordre de l'être mais d'une relation qui exige effectuation. La définition du caractère $l i$ (rite) qu'on trouve dans le Shuowen jiezi me semble être rédigée en écho. Nous en avons déjà mentionné la première partie ; citons-la ici toute entière : «Rite : mise en œuvre. Ce par quoi on sert les esprits pour en obtenir bénédictions. " (Comme le souligne plusieurs fois Gilles Boileau, la première de ces bénédictions est la viande issue des sacrifices, dont elle constitue le produit.) Commentant cette définition, Gilles Boileau, fidèle à sa perspective, parle ici de " deux choses qu'il faut distinguer soigneusement » (i.e. la mise en œuvre d'une part, le service des esprits de l'autre, p. 422). C'est donc après l'analyse de ce que recouvre la définition du rite comme sa pratique même qu'il se heurte au problème de "l'expérience religieuse " qu'il autorise, et notamment du type d'expérience évoqué par la citation de Confucius analysée plus haut. Sans qu'il l'énonce directement, la thèse implicite est celle d'une sécularisation progressive du rituel, qui en quelque sorte échapperait à la gangue $\mathrm{du}$ religieux pour déployer pleinement ses effets sociaux : "Les rites sont motivés au principe par une intentionnalité religieuse mais dans un second temps informent également les règles de comportements sociaux admis non directement liés à la pratique religieuse. » (p. 427)

J'ai déjà évoqué plusieurs raisons ou indices qui me font questionner sinon la véracité du moins la pertinence opérationnelle de ce schéma. Il me paraît ne 
pas faire justice à la problématique de Confucius et à celle (multiforme) de ses successeurs : les uns et les autres centrent effectivement leur réflexion sur les racines et la culture d'une intentionnalité, mais, chez Confucius, cette dernière se caractérise par le fait qu'elle ordonne et régit l'ensemble des domaines de la vie humaine (une preuve, parmi bien d'autres, réside dans le fait qu'il affirme explicitement que celui qui ne sait comment servir les hommes ne saurait servir les esprits, cf. Analectes 11, 12). Lorsque les soubassements anthropologiques et/ou les effets sociaux de l'intentionnalité confucéenne et de son inscription rituelle sont mis en question (par les auteurs taoïstes, mohistes ou légistes respectivement), le rite ne fait pas l'objet d'une opération de "sécularisation " mais est au contraire intégré dans une cosmologie plus développée qu'en ses débuts, en même temps qu'il est parfois (pas toujours) contenu dans le domaine du pur " religieux » (que sa relégation même institue), l'ordre sociopolitique étant régi alors principalement par des moyens qui n'appartiennent plus au domaine du rituel. Et la reprise confucéenne de l'époque Han dont témoigne le Shuowen jiezi définit le rite, en un tout indissociable, comme mise en ouvre et service des esprits producteur de bénédictions.

\section{L'exercice du rituel, entre intelligence et sagesse}

Deux observations complémentaires peuvent éclairer les directions d'enquête que je suggère ici, lesquelles visent à relier (sans pour autant confondre) l'analyse de l'expérience rituelle avec celle de son institution et des changements qui l'affectent. La première concerne l'importance accordée par Confucius et la tradition qu'il inaugure à ce que je me risque à désigner comme la vertu d' " attention pure ». Les interprétations canoniques données de l'hexagramme vingt du Yijing (guan: observation, contemplation) en font le moment du rituel où l'officiel suspend son acte, tout pénétré de ce qu'il fait: cette suspension, ce vide, cet intervalle non seulement sont partie du rituel même, mais encore s'avèrent indispensables pour lui conférer sens et efficace. L'investissement intérieur dont il est ici question est également exprimé par le caractère jing (traduit souvent par respect) mais aussi par le propos dans lequel Confucius déclare que savoir une chose ne vaut pas le fait de l'aimer, et que l'aimer ne vaut pas le fait d'en faire sa joie (Analectes 6, 20). Il trouve son expression la plus forte dans le propos lequel Confucius, interrogé sur le moyen de développer en son intérieur la vertu d'humanité répond: "Ne rien voir hors du rite, ne rien entendre hors du rite, ne rien dire hors du rite, ne rien faire hors du rite. » (Analectes 12,1) En d'autres termes, c'est la pratique rituelle même qui est acte d'attention pure, applicable à tout aspect de l'existence. Cette qualité d'attention, notons-le bien, n'est en rien figée, compassée : l'équilibre à maintenir entre culture et naturel est un autre thème récurrent des Analectes (voir par exemple 7, 18 et 10, 27).

La deuxième observation est éveillée par une réserve que j'entretiens sur un choix de traduction effectué par Gilles Boileau (hâtons-nous de noter que ses 
traductions sont d'ordinaire remarquables et fort éclairantes, d'autant qu'elles se mesurent à des textes parfois très difficiles; mais comme tout un chacun, il effectue parfois des choix qui découlent de parti-pris). Dans le chapitre 5, consacré à la montée des shi, l'A. fait un large usage de la notion d' "intelligence ", dont il rapproche la formation en Chine ancienne de l'apparition du concept d'intellection dans le vocabulaire d'Aristote. Le thème est d'importance pour notre sujet, puisque l'usage de l'intelligence va transformer les fonctions du rituel en modifiant le rapport au réel au travers de la possibilité donnée au pouvoir de "prescrire la réalité en la nommant» (p. 363). Une difficulté réside dans le fait que, dans ce chapitre, l'A. traduit par "intelligence » toute une gamme de caractères chinois : p. 320, c'est le caractère xin (cœur/esprit) qui est ainsi rendu, ce que le contexte du passage ne corrobore pas, puisqu'il s'agit de ministres qui seraient ou ne seraient pas "d'un seul cœur et d'un même esprit ». Dans la traduction offerte p. 326 d'un fameux passage des Analectes $(6,23)$ qui attribue à l'homme sage la joie et l'amour de l'eau, et à l'homme d'humanité la longévité et l'amour de la montagne, c'est le caractère zhi (connaissance - traditionnellement assimilé ici à son homonyme signifiant sagesse) qui est traduit par intelligence. La traduction est moins problématique, en ceci que le texte attribue bien à l'homme sage l'ondoyance aquatique que Gilles Boileau assigne à l'intelligence déployée par les shi, mais elle est loin de rendre compte de l'entièreté du sens du passage, lequel n'oppose pas, mais bien plutôt associe au sein d'un même "paysage mental ", les vertus de sagesse et d'humanité. Aux pages 335-336, dans un passage du Hanfeizi, c'est le caractère $z h i$ désignant la sagesse qui est rendu par intelligence, une traduction qui est ici tout à fait légitime, puisqu'en certains cas ce caractère désigne spécifiquement la connaissance de "stratagèmes ». Mais l'acception diffère autant qu'il se peut de celle qu'on trouve en Confucius : alors que Hanfeizi oppose l'homme intelligent à l'homme moral (recommandant du reste de choisir le second comme ministre), pour Confucius on ne saurait être homme sage ou bien homme empli de la vertu d'humanité, pas plus qu'on ne saurait séparer les eaux de la montagne dans le paysage qui les assemble. Enfin, il est plus difficile d'accepter le fait que, pour divers passages du Zhuangzi, les termes de zhi (connaissance) et xin (cœur/esprit) soient uniformément traduits par «intelligence» (p. 338-343).

Qu'on nous entende bien : le rendu des passages est suggestif, et, pris isolément, tous ces choix sont justifiables. La difficulté réside en ceci que la gamme des attitudes psychologiques, morales et même spirituelles ouverte par ces textes est réduite d'autant, au profit de l'accent porté sur l'ondoyante stratégie des shi, la classe porteuse des transformations politiques et rituelles. Or, l'audace que l'A. reconnaît à un Mencius par exemple (p. 372) s'exprime aussi par l'invention de la «sagesse » $(z h i)$, une catégorie que l'accent ici porté sur ce construit qu'est «l'intelligence » me semble avoir pour mission de déconstruire par défaut. Aux antipodes de l'intelligence ondoyante, il y a des accents sotériologiques chez 
Mencius : «La vertu d'humanité, c'est le cœur de l'homme; et la justice [la droiture], c'est sa route. Plaignez l'homme qui a abandonné la route et ne la poursuit pas, qui a perdu son propre cœur et ne sait où la trouver. Quand on perd son chien ou ses volailles, on sait par où aller les chercher. Quand on a perdu son cœur, on ne sait pas vers où aller. La voie de l'apprentissage, ce n'est rien d'autre que d'aller à la recherche du cœur perdu - et c'est tout. » (Mencius 6 A 11) (Je traduis ici xuwen par " apprentissage » parce que la leçon même du passage semble induire que l'étude ne pourra s'effectuer que sous la conduite d'un maître, lequel orientera le disciple sur la voie de ce qu'il avait perdu et ne saurait retrouver par lui-même.) Les options morales et spirituelles de Mencius sont en rapport étroit avec sa conception du rituel : sommé de déclarer si le rite est plus important que la nourriture ou le sexe, il demande si la faim entraînerait son contradicteur à tordre le bras de son frère pour lui prendre par force sa subsistance, ou l'appétit sexuel à grimper par dessus le mur du voisin pour s'approprier sa fille, liant une fois encore rituel et vertu d'humanité en une synthèse qu'il se refuse à desserrer (Mencius 6 B 1).

Le point essentiel est ceci : la réflexion menée sur le rituel durant les Printemps et Automnes puis les Royaumes combattants (de la fin des Printemps et Automnes à l'avènement de la dynastie Qin en 221 av. J.-C.), réflexion qui entremêle considérations politiques, morales, religieuses et spirituelles, n'est pas déterminée simplement par la raison calculatrice que développeraient les shi, mais par le débat entre écoles qui fait de la place et de la portée du rituel un enjeu toujours disputé. Dans l'histoire de la Chine, c'est la position de Mencius qui - intellectuellement du moins - l'emportera, même s'il faudra attendre l'époque du confucianisme Song pour que sa victoire s'affirme.

Il y a donc bien un "moment axial » de la pensée chinoise, que la montée en puissance des shi peut expliquer pour partie, mais qu'on ne saurait résumer à leur ascension (l'analyse comparée de ce «moment axial » menée par Robert Bellah dans Religion in Human Evolution, 2011 - notamment dans son chapitre 8 - me semble exemplaire et de prudence et de justesse). Avant les shi, l'ordonnance rituelle servait sans nul doute à assurer, évaluer et représenter celle du social et du politique; mais l'interprétation confucéenne du rituel focalise son objectif et son efficace vers l'ordonnance intérieure qu'il produit. S'affirme là une nouveauté, qu'il faut accepter de penser comme telle, même si la nouveauté se présente préférentiellement sous la forme d'une réinterprétation des usages des temps passés. Certes, en un sens l'ancrage du rituel dans la conscience morale du sujet peut renforcer encore la manipulation des rites aux fins de contrôle politique ; mais, droitement opéré et compris, pareil ancrage fait bien du rituel le lieu d'un discernement qui surplombe l'ordre politique.

Le paradoxe, c'est que, telle que présentée par Confucius et certains textes postérieurs, la pratique rituelle à la fois ordonne et libère. Nous l'avons déjà noté en citant la phrase de Fingarette qui compare la performance rituelle à 
l'exécution musicale, l'un et l'autre révélant le degré de sincérité et de liberté intérieure de l'exécutant. L'obtention de la liberté intérieure que produisent l'étude et la pratique est illustrée par l'évolution personnelle de Confucius telle qu'il la relate (Analectes 2, 4). L'attention à pareille dimension peut nous aider à relativiser l'opposition entre les textes taoïstes et confucéens : le fameux apologue du Zhuangzi sur le Boucher Ding, sacrificateur dansant devenu à tel point expert dans le maniement du couteau que jamais ce dernier ne s'émousse (Zhuangzi, chapitre 3), est manifestement écrit par un spécialiste du rituel. L'éloge du Naturel qu'il poursuit subvertit sans doute l'ordre qu'il est censé assurer (du reste, à prendre ce seul passage même cette conclusion n'est pas entièrement assurée), mais cette subversion est toujours ancrée dans la fidélité à une pratique qui se révèle libératrice. (Les références musicales de l'extrait discutées par Boileau p. 85-86 ne me semblent en rien parodiques comme il le voudrait : elles insèrent l'acte du sacrificateur dans le contexte du surgissement de la vie réalisé par l'opération du yin-yang. Par ailleurs, l'une des leçons du texte est bien de lier pratique rituelle et vide du cœur, leçon acceptable bien au-delà de l'école taoïste.) De même, lorsque Zhuangzi se met à croupetons et chante en frappant sur un pot lors du trépas de son épouse (chapitre 18), c'est un « contre-rituel » qu'il accomplit, et un contre-rituel reste toujours un rite, même si ce dernier détourne le sens de la performance que l'on était en droit d'attendre. Le rôle ambigu que, de bout en bout, le texte du Zhuangzi fait jouer à Confucius pourrait en fournir d'autres preuves : si les penseurs des Printemps et Automnes se querellent sur la façon dont la pratique des rites (des "propriétés " comme on traduit parfois) intègre et développe ordre et liberté dans l'intérieur de l'individu qui s'y livre, c'est parce qu'ils ont collectivement ouvert l'espace d'un rapport dialectique entre le premier et la seconde. À partir de ce moment, le rapport entre " rituel » et " politique " ne saurait se penser sans référence à un troisième terme : celui de «l'homme profond " (comme on traduit parfois le terme confucéen de junzi - rendu encore par gentleman homme de bien ou honnête homme), qui pratique et régule l'un et l'autre depuis le lieu de son intériorité et de son jugement moral.

Revenons ici aux observations d'Erica Bradley sur le caractère relativement tardif des développements sur le caractère proprement cosmique de la musique rituelle : on peut se demander s'il ne fallait pas cet ancrage de la pratique rituelle dans l'ordre de l'intériorité pour qu'au microcosme de l'individu réponde le macrocosme cosmique qu'ordonne et unifie la musique des sphères comme la musique rituelle ordonne et unifie le monde moral. Un indice de cette liaison serait le fait que, pour Mencius - l'un des premiers sinon le premier à théoriser le terme - le qi (l'énergie) est d'abord une qualité morale (Mencius II A 2), et c'est plus tard que le terme prendra les accents cosmiques qui lui sont désormais associés.

Pour nous résumer : "l'intentionnalité religieuse » du rituel évolue au fil du temps, comme fait son intentionnalité politique, et les synthèses qui marquent 
à divers moments l'inscription des notions et pratiques qui lui sont associées dans les mécanismes fondateurs du social restent indissociablement et politiques et religieuses. L'une des dimensions ne diminue pas au profit de l'autre : c'est plutôt une élaboration, une synthèse nouvelle qu'appellent à chaque fois les moments disjonctifs de l'évolution sociale et intellectuelle.

\section{Rituel, nature et culture}

Xunzi (un contemporain de Mencius, quoique probablement beaucoup plus jeune que lui - les dates sont incertaines) est sans doute le penseur de la Chine antique (ou même de toute l'histoire chinoise) qui a développé la réflexion la plus approfondie sur la nature et les fonctions du rite - une réflexion difficile à saisir parce qu'elle part d'une approche sophistiquée de la nature et du social, une approche qui ne saurait se résumer à ce que l'on croit connaître de sa thèse selon laquelle la nature humaine (xing) est " mauvaise » $(e-$ un caractère qui a la connotation de «féroce » ou "vicieux »). Ce qui nous importe ici, c'est que «le Xunzi annonce la prééminence, au début de l'empire sous les Han, d'un confucianisme mâtiné de légisme qui l'emporte sur l'inspiration mencienne " (Anne Cheng, Histoire de la pensée chinoise, Paris, Le Seuil, 1997, p. 231). Tout en présentant une version fonctionnaliste de l'origine des rites, l'œuvre intègre en un tout les différentes approches du rituel (spirituelles, politiques et cosmiques) évoquées dans les pages qui précèdent : «Les rites ont trois fondements : le Ciel et la Terre sont le fondement de l'engendrement, les ancêtres le fondement de l'espèce, les souverains et les maîtres le fondement de l'ordre. [...] Par les rites [l'homme] sert en haut le Ciel, en bas la Terre, il honore ses ancêtres et exalte son souverain et son maître : tels sont les trois fondements des rites. » (cit. in Anne Cheng, op. cit., p. 229).

J'introduis ici l'ouvrage de Hong cité en bibliographie, d'abord parce qu'il présente un excellent exemple de l'herméneutique des textes anciens entreprise par une nouvelle génération de chercheurs chinois, ensuite parce qu'en se focalisant sur le rapport entre " art du cœur » (xinshu) et " voie d'ordonnancement " (zhidao) chez Xunzi il rejoint exactement notre sujet. Le point de départ est le suivant: les penseurs chinois se sont régulièrement demandé comment Xunzi, puisqu'il postule la nature humaine mauvaise, pouvait attribuer aux Sages des temps anciens, dotés après tout de la même nature que les autres hommes, la création des rituels. Pour l'A. cependant, Xunzi implique qu'il était simplement nécessaire que soient créés les rites et le sens des propriétés (ou de la justice) qui les accompagne, cela parce qu'il n'était pas d'autre façon d'éviter l'écroulement complet de l'ordre humain et naturel. Un mode de raisonnement, observe Hong, très proche de celui que Hobbes développera bien plus tard. Cela ne fait pas de Xunzi un Légiste : la nature peut toujours être contrôlée, transformée, en quelque sorte façonnée à neuf par la fabrication que sont justement les rites et les instructions doctrinales. 
Le "pont à établir entre la nature humaine, mauvaise, et le rituel, bon " (Hong, p. 14) le sera par le truchement d'une réflexion approfondie sur le xin (cœur/esprit), lequel arbitre entre les diverses passions (qing) qui expriment les tendances à l'œuvre dans la nature humaine (xing). En d'autres termes, Xunzi définit le cœur/esprit comme la capacité à arbitrer entre bonnes et mauvaises inclinaisons, tandis que les organes des sens mettent ces dernières en action. Xin est donc le maître de la personne, le donneur d'ordres, le point de passage obligé entre l'intérieur et l'extérieur. La pratique de l'étude vise à contrôler la façon dont le cœur connaît et par la suite ordonne. Si nombre de penseurs ont critiqué l'artificialité de la pensée de Xunzi, ce dernier semble revendiquer cette même artificialité, seule façon d'éduquer et conduire des individus qui vivront de telle façon à rendre possible un monde vivable alors même que leur nature est soumise aux passions. À cette artificialité, note l'A., s'ajoute une hiérarchisation précise des niveaux culturels et spirituels atteints respectivement par le Lettré (shi), le gentleman (junzi) et le Saint (shengren), ce dernier seul capable des surmonter pleinement l'opposition de départ entre dispositions internes et observance externe.

La façon dont Xunzi organise le gouvernement de l'extérieur et de l'intérieur, observe encore l'A., semble comporter un élément yang et un élément yin: le premier consiste dans le gouvernement d'autrui au moyen des rites; le second dans le gouvernement de son propre cœur en travaillant à y faire régner la "vacuité » $(x u)$. Autrement dit, la pensée de Xunzi réaliserait une synthèse entre les écoles taoïstes et Huang-Lao d'une part, lorsqu'il s'agit de l'éducation de l'intériorité, et des principes confucéens et pré-légistes de l'autre lorsqu'il est question de gouvernance politique. La première partie de l'enseignement est centrée sur la connaissance de "l'imperceptible", du caché (wei) tandis que la seconde se mesure avec la gestion de la prudence et de la peur (un caractère wei homonyme). Ce balancement incessant est certainement typique du style de pensée de Xunzi. Cette synthèse allait influencer en profondeur les penseurs de la transition dans laquelle la Chine allait s'embarquer juste après son décès, lequel survint vraisemblablement quelques années avant l'avènement des Qin.

\section{Dysfonctionnement et anxiété}

La façon dont Xunzi lie étroitement la nécessité du rite et son artificialité foncière peut nous éclairer sur l'anxiété qui pouvait saisir les ritualistes à penser tant la nature de leur activité que les difficultés rencontrées dans sa pratique. C'est cette anxiété qui est au cœur du livre de Michael Ing. Son titre résume clairement son objet: The Dysfunction of Ritual in Early Confucianism. Au travers d'une lecture du Liji c'est la question des différentes manières de se mesurer aux « échecs » du rituel dont il est ici question. La perspective ainsi apportée au "Mémoire sur les Rites " diffère quelque peu de celle de Boileau puisqu'il s'agit ici d'une lecture herméneutique d'un ouvrage donné, non d'une enquête croisée sur l'évolution des pratiques rituelles. 
Résumant sa lecture du Liji, l'A. comprend par rituel toute "performance scriptée (scripted performance) visant à ordonner le monde - le monde étant compris comme incluant des phénomènes sociaux comme les relations mâlefemelle, des réalités telles que les dispositions intérieures d'un être humain, des choses non humaines comme la pluie ou les pouvoirs spirituels » (p. 18). Les spécialistes de la Chine antique, note l'A., ont développé des approches plus ou moins restrictives ou extensives de ce que recouvrait le terme. Pines et Sato ont relevé l'élargissement régulier des réalités recouvertes par le terme. Roger Ames a insisté sur le fait que le " rite » ne se réduisait pas aux rituels mais recouvrait assez vite une qualité d'attention et de présence à toute réalité de la vie ordinaire. William Theodore de Bary a montré que l'extension progressive du sens d'un terme était une tendance à l'œuvre dans l'ensemble du confucianisme originel : l'importance d'une notion ne se traduisait pas par le fait de la définir plus précisément mais bien plutôt par celui d'en élargir, assouplir l'usage. Le terme li ne fait pas exception à la règle. Remarquables à cet égard sont les citations prises du chapitre Zhongni yanju, lequel fait parler Confucius : "Pour le junzi, rien n'est qui ne soit rituel. [...] Dire [une chose] et la mettre en œuvre [lü], voilà le rite ; faire [une chose] et y prendre joie, voilà la musique. " Ing prête aussi une attention bienvenue à l'importance de la métaphore aquatique pour la compréhension du rituel dans le Liji (importance qui rapproche ce livre du Mencius par exemple). Comme le fait une digue, le rite à la fois canalise les dispositions des agents rituels et autorise leur expression (p. 19).

En même temps, l'apport de l'A. est de montrer que le Liji (ouvrage composite dont la date présumée de composition, le style et les préoccupations varient de chapitre en chapitre) comprend bien diverses acceptions du $l i$, parfois restrictives, parfois extensives. Autrement dit, le caractère désigne quelquefois des " rituels ", quelquefois une attitude d'ensemble. Il n'en existe pas moins des marques de repère propres à l'ouvrage : les rituels sont décrits au début du chapitre Liyun comme des $j i$ (des "nœuds commémoratifs " traduit l'auteur; c'est exact mais ne rend pas tout à fait justice à l'histoire d'un caractère compliqué qui possède le sens important de "règles", de fil conducteur, de démarrage d'un mois lunaire, et qui finit par prendre le sens paradoxal de démêlage des fils de soie). L'idée qu'on ordonnance le monde au travers de "nœuds ", qui assurent les rapports entre temps, espaces, phénomènes, est à même de regrouper un nombre assez large d'acceptions.

L'A. voit les « échecs » enregistrés dans la performance rituelle comme une source d'anxiété qui alimente nombre des discussions du Liji - anxiété redoublée par la difficulté de distinguer entre les échecs qui auraient pu être évités et ceux qui étaient inévitables. Distinction qui en recoupe une autre sans s'y identifier : celle qui oppose l'erreur dans l'exécution du «script» régissant le rituel (failure in competency) et l'échec provoqué par le fait que la situation était trop complexe pour que le rituel y réponde adéquatement (failure in efficacy). Dans ce dernier 
cas, reste la possibilité que l'agent en charge du rituel soit capable d'en altérer le script pour en éviter l'échec. Les échecs vraiment inévitables sont donc ceux qu'aucun agent ne saurait prévenir - tels ceux causés par un incendie soudain dans le hall ancestral. Les situations ambiguës sont illustrées par la tombée soudaine de la pluie détruisant la tombe édifiée par Confucius pour ses parents : difficile de décider s'il faut imputer cet échec à la pure malchance ou au fait que Confucius construisait une tombe plus solennelle que ne le demandaient les coutumes anciennes. Le rituel est donc affecté par une tension entre son but avoué - édifier, maintenir un ordre - et le fait qu'il est par essence une entreprise risquée, soumise à divers aléas.

À ce point, Ing revient sur la relation entre le $l i$ et le $y i$, ce deuxième caractère étant traduit le plus souvent par "justice » ou " propriété », mais détenant aussi le sens de "caractère approprié " (à une situation donnée). Pour des auteurs tels Robert Eno et D. C. Lau, le $y i$ interprète et oriente le li. Un cas d'école est donné par Mencius : même si les exigences rituelles interdisent qu'un homme et sa belle sœur se touchent, il est requis de venir au secours de sa belle sœur en passe de se noyer (Mencius $4 \mathrm{~A}$ 17). On comprend que le terme $y i$ en vienne à signifier le "sens " de cela qui est fait: le rite ne fait sens qu'en fonction du contexte dans lequel il est mis en œuvre. Cette position n'est pas la seule tenable: des logiciens tels Bryan Van Norden (Virtue Ethics and Consequentialism in Early Chinese Philosophy, 2007) montrent que l'interprétation (ruling) ne saurait être incluse dans la règle (rule), laquelle ne peut donc être que provisoirement suspendue. La plupart des discussions autour du rite comme "systèmes de règles ", note l'A., sont influencées par deux tendances: celle de traiter le rite comme une "grammaire culturelle ", et la volonté contemporaine de faire des auteurs confucéens avant tout des penseurs de l'éthique, dans la ligne du renouveau des "éthiques de la vertu» (p. 65-68). On ne voit cependant pas comment Ing trace une voie alternative, sinon peut-être en insistant sur l'importance pour les auteurs du Liji de la question de l'efficace. L'échec du rituel, note-t-il encore, constitue aussi son ouverture : c'est par lui qu'il est permis au rituel d'être transformé et que s'ouvre l'espace d'une quête continuée de ce qu'exige une vie sensée (p. 218).

Si Boileau se tient loin des ritual studies, Ing y est à ce point immergé que la pertinence de son questionnement s'en ressent parfois : chez lui, le terme "rituel » est tellement prégnant que celui de « sacrifice » n'apparaît même pas dans l'index. Autant l'angle de "l'anxiété" du spécialiste rituel me semble excellent pour mieux approcher «ce que fait » le rituel en Chine antique, autant je ne crois pas que cette anxiété puisse être expliquée en termes de moyen et de but (un rituel donné visant à assurer une fin donnée). Le projet même d'ordonner le monde, et le rapport (tendu) entre $j i$ (sacrifice) et $l i$ (rituel) que ce projet implique, voilà, me semble-t-il, ce qui met en branle la spéculation des ritualistes. Je reviens sur ces points dans la section finale. 


\section{Rituel et divination}

J'ai hésité à insérer une présentation du livre de Léon Vandermeersch dans la discussion suscitée par le livre de Gilles Boileau. Qui parcourt l'un et l'autre de ces ouvrages aura sans doute l'impression de se trouver dans deux univers étrangers l'un à l'autre, alors même que tous deux traitent bien des mêmes temps et lieux : chez Boileau, un monde composé de femmes ou d'infirmes exposés au bûcher, d'ébats sexuels dans des champs de mûriers, de festins et d'ivresses, de Lettrés rusés et querelleurs. Chez Vandermeersch, un univers ordonné, composé d' " équations divinatoires ", de " rationalisation lexicale ", de "culture manticologique ». La différence de style tient pour partie à la différence des sujets : approche anthropologique du rôle tenu par la pratique rituelle pour assurer une cohérence sociale toujours menacée d'éclatement chez Boileau ; pour Vandermeersch, étude des sources divinatoires et graphiques d'une épistémè dont certains pans subsistent jusqu'à aujourd'hui.

Le livre de ce dernier s'offre comme une synthèse brève et puissante des conclusions atteintes par un maître de la discipline. Nombre des aspects de l'étude sont en dehors du sujet de cette note, et je ne les aborde donc pas. Et il vaudra mieux relativiser le sens et la portée de la phrase qui ouvre ce livre : «La profonde différence entre la culture occidentale, qui divergent l'une de l'autre d'autant que l'écriture idéographique s'éloigne de l'écriture alphabétique, a son origine dans l'opposition entre, en milieu chinois, une pensée primitivement guidée par une forme très sophistiquée de divination, et, en milieu gréco-latin et judéo-chrétien, une pensée primitivement guidée par des croyances religieuses » (p. 9). Sans réduire le rien du monde, le rôle joué par la mantique dans l'apparition de l'écriture chinoise, ni celle de cette dernière dans un système épistémique et cosmologique d'ensemble, il est permis de (a) ne pas vouloir dissocier la forme de divination née en Chine d'un système religieux d'ensemble ; (b) noter que les croyances religieuses qui sont ainsi globalement contrastées avec le système chinois sont elles aussi marquées à l'origine par la prévalence des pratiques et spéculations divinatoires (même si le matériel divinatoire et le mode de notation des résultats des consultations jouent de fait un rôle stratégique dans la bifurcation culturelle qui s'opère) ; (c) se demander quelles sont la validité et la portée opératoire d'un binôme opposant « divination » à " croyances religieuses ». (Une comparaison d'ensemble de la « raison divinatoire » en monde chinois et en monde gréco-romain par exemple reste à conduire. Sur ce dossier, on pourra se référer à plusieurs contributions de Lisa Raphals, mais aussi aux recherches en histoire comparée des sciences antiques de Geoffrey Lloyd et Nathan Sivin.) Mais une fois encore il est permis de relativiser le constat sur lequel s'ouvre l'ouvrage, et de le lire simplement comme une magistrale synthèse sur la façon dont la divination pratiquée sur omoplates de bœuf et carapace de tortue a généré tout à la fois la création d'une "langue graphique » et celle d'une "raison divinatoire ", lesquelles structurent tous les aspects de la pensée chinoise. 
Contrairement à Boileau, Léon Vandermeersch insiste sur l'importance du rapport entre rite et musique, et émet d'intéressantes observations sur l'origine des hymnes rituels. Observations insérées dans des développements qui soulignent les origines divinatoires des élaborations rituelles : "Il y a tout lieu de penser que la parenté de la fonction de scribe-devin avec celle de musicographe remonte à la parenté de la fonction de scapulomancien avec celle de chaman. En même temps que la divination devenait science manticologique et que la religion devenait système de rites, les anciennes danses chamaniques se sont transformées en un rituel de danses et de chants du "Canon des Odes", rituel qui a hérité de son prototype de transe envoûtante une force d'empathie qu'exploite le ritualisme, de même que la langue graphique a hérité de son prototype divinatoire une puissance herméneutique qu'exploite l'exégèse canonique. » (p. 62-63) Point n'est besoin de cet appel assez contestable à la transe pour souligner simplement ceci : au départ, le rituel (y compris dans son aspect sacrificiel, qui est l'objet de la partie suivante) n'est sans doute pas séparable d'un processus divinatoire. L'évolution de la notation manticologique en un système graphique d'ensemble libère pour grande part la «raison divinatoire " de son armature rituelle, en même temps qu'elle autorise une évolution du sens des comportements rituels comme de celui des effets personnels et sociaux qu'ils produisent. Et l'on pourrait alors faire l'hypothèse d'un parallélisme entre "spéculation divinatoire » et "spéculation rituelle ", la première portant sur la structure même du cosmos, la seconde sur la façon proprement humaine de s’y insérer. Poursuivant sur la voie tracée par Vandermeersch, on pourrait alors suggérer que la construction rituelle est tout autant déterminée par une "rationalité formelle » (au sens wébérien du terme) que l'est la construction manticologique.

\section{Et le sacrifice?}

Un terme n'apparaît guère dans le monde ordonné tracé par l'ouvrage de Léon Vandermeersch. C'est celui de "sacrifice "- alors même que l'auteur de La Voie royale a notamment donné une description classique du grand Sacrifice au Ciel dans la banlieue sud (La Voie royale, 1980, t. II, p. 355-371). La notion surgit, certes, mais tout se passe comme si un développement indépendant sur la matérialité du sacrifice risquait de briser le souverain équilibre du monde mental et social ici décrit. Ce silence n'est en rien nouveau, et reflète pour bonne part celui des auteurs chinois anciens eux-mêmes. Pourtant, la prévalence des sacrifices humains est attestée par l'archéologie. Pour la période finale de la dynastie Shang (phase Anyang, environ 1250-1050 avant notre ère), le nombre de fosses sacrificielles et tombes annexes découvertes dans la métropole royale s'élève à plus d'un millier (les fosses recèlent les restes des prisonniers de guerre et autres victimes, guère distinctes des animaux sacrifiés; les tombes annexes abritent les «gardiens » de la tombe principale). Les fouilles de sites datant des Zhou occidentaux témoignent d'une raréfaction de la pratique des sacrifices 
humains ( $c f$. Alain Thote, "Les pratiques funéraires Shang et Zhou, interprétation des vestiges matériels ", in John Lagerwey, dir. Religion et société en Chine ancienne et médiévale, notamment p. 49-58). Beaucoup plus tard, sous la dynastie des Han occidentaux, les sacrifices humains ont en général disparu mais l'ampleur des sacrifices à la terre, au Ciel et aux esprits locaux est telle que durant le règne de l'Empereur Ai (7-1 avant notre ère) on recense trente sept mille sacrifices par an répartis sur plus de sept cents lieux de culte (Marianne Bujard, "Cultes d'État et cultes locaux dans la religion des Han ", in John Lagerwey, ibid., p. 319). C'est sur cet arrière-plan d'épuisement de l'efficace sacrificielle qu'il faut comprendre le surgissement du taoïsme et du bouddhisme - concomitant à l'écroulement des Han - comme religions anti-sacrificielles. "Les prêtres de la nouvelle religion n'entrèrent pas en communication avec les hiérarchies surnaturelles en s'adonnant à des rites sacrificiels [...] mais au moyen d'un échange épistolaire, c'est-à-dire à une correspondance écrite soumise à d'innombrables règles bureaucratiques, la guérison des malades par exemple reposant sur la confession des péchés et des pétitions adressées aux ministères divins appropriés. " (Anna Seidel, "Taoïsme : religion non officielle de la Chine ", Cahiers d'Extrême-Asie, VIII, 1995, p. 16.)

Alors que les pratiques sacrificielles, y compris de sacrifices humains, sont partout avérées, elles ne semblent pas explicitement « réfléchies » par les penseurs chinois anciens. Notons tout de même la réprobation progressive exprimée par certains des auteurs cités à l'encontre du sacrifice de $w u$ femmes ou d'infirmes, réprobation inséparable d'une volonté de moralisation du Rite comme tel (voir Boileau, p. 50-51). Mais Confucius lui-même reste un défenseur des anciennes pratiques : "Zigong voulait supprimer le sacrifice du mouton dans le rituel de l'Annonce de la nouvelle lune. Confucius dit: "Ah, tu tiens à tes moutons ; moi, je tiens à mes rituels !” "(Analectes 3, 18) C’est pareil contexte qui explique la position de Jean-François Billeter : «Les sacrifices [...] manifestent sur le plan religieux le monopole de la violence que l'aristocratie exerce en s'adonnant à la guerre et à la chasse. Pour codifiée et ritualisée que soit cette violence elle n'en est pas moins absolue et culmine dans le sacrifice humain que l'on continue à pratiquer sporadiquement au temps de Confucius [...]. Les ritualistes qui se réclameront de Confucius défendront l'idée qu'aux morts, qui sont à la fois absents et présents, il convient de faire des sacrifices absents et présents, c'est-à-dire symboliques. Jean Lévi montre que cette position est restée mal comprise et que la crainte de ne pas assez servir les morts incitait à revenir aux sacrifices réels. Il n'y a donc jamais eu de rupture. Le sacrifice humain n'a jamais été condamné. Il a été occulté, il est devenu l'inavouable "scène primordiale" cachée au cœur de la tradition confucianiste. [...] Le confucianisme ancien est resté prisonnier de ce sacré refoulé. » ("L'énigme Confucius », Esprit, aoûtseptembre 2003, p. 78-79.)

On peut se remémorer ici les observations du grand indianiste J. C. Heesterman, lequel notait en 1993 que, dans les études religieuses, le terme "sacrifice » avait 
presque entièrement disparu au profit de celui de " rituel », et que ce glissement permettait de substituer aux questions sur le «sens" des sacrifices offerts des considérations sur les «structures » des jeux rituels (The Broken World of Sacrifice. An Essay in Ancient Indian Ritual). On ne saurait faire ce reproche à Gilles Boileau, lequel fait largement usage de la catégorie de sacrifice, et ne se voile certainement pas la face devant la "scène primordiale " du ritualisme chinois. Le chapitre 2 de son ouvrage met en lumière le fait que le corps du roi est potentiellement matière sacrificielle, qu'un système de substitution permet d'épargner (voir notamment p.129-132). Le chapitre 3 est spécifiquement dédié, comme on s'en souviendra, à la " cuisine du sacrifice. " Je lui reprocherais néanmoins d'être trop rapide sur la distinction à opérer entre $l i$ (rituel) et $j i$ (sacrifice - ne pas confondre avec le $j i$ homonyme de " nœuds divinatoires »). Même s'il est vrai que les textes classiques semblent parfois utiliser ces deux caractères (presque) indifféremment (je crois en fait que ce sont nos grilles de lecture qui nous rendent trop peu alertes aux accents impliqués), il n'est pas indifférent que la graphie de ji représente très explicitement une main saisissant un morceau de viande présenté aux esprits, tandis que celle utilisée pour li dessine un brouet de céréales déposé dans une coupe (avec apposition tardive du radical signifiant spirituel, esprits ou choses sacrées). C'est donc le caractère désignant l'offrande végétale qui a fini par désigner en Chine l'ensemble des conduites rituelles, parmi lesquelles celles gouvernant les sacrifices sanglants.

Le faible intérêt déployé par Gilles Boileau quant aux distinctions à opérer entre rituel et sacrifice s'explique par l'attention prioritaire qu'il porte aux modalités du traitement et de la répartition des viandes produites par l'opération sacrificielle, modalités qui gouvernent pour lui tant la compréhension par les participants du processus historique d'humanisation que la nature des relations sociales (réciprocité et hiérarchie). C'est même, comme nous l'avons vu, la prédominance de ce motif qui fait l'originalité de son approche. En d'autres termes, dans les trois éléments qui composent un sacrifice - exécution, destruction, distribution -, il privilégie fortement la distribution. Reste la réflexion de Heesterman : tout rituel n'est pas sacrifice bien entendu, mais encore tout dans le sacrifice n'est pas rituel (Heesterman voit dans le sacrifice une "énigme » portant sur la relation entre vie et mort, énigme autour de laquelle toutes affaires humaines et extra humaines se décident, énigme dont le rituel rejoue sans cesse les termes sans jamais pour autant la résoudre). La piste privilégiée par Gilles Boileau l'amène donc à occulter certains aspects du sacrifice. Il note ainsi juste en passant des témoignages de sacrifices d'holocauste : «On brûle [une victime] au Ciel sur le grand monticule. On enterre [une victime dédiée] à la terre près du grand monticule [de terre]. Les victimes sont des veaux à la robe rousse. » (Liji, chapitre Jifa, cit. p. 154 ; voir aussi p. 104 et suiv.). Et pourtant, ces indices et plusieurs autres montrent que le sens du sacrifice ne s'arrête pas à la production des viandes à partager. Sur cette seule base, il serait impossible de comprendre la 
grande crise sacrificielle qui va frapper la religiosité chinoise durant la dynastie Han. Si cette dernière suit immédiatement la période sur lequel le livre de Gilles Boileau se clôt, c'est bien sous les Han que nombre de conflits et d'apories décrits par son ouvrage trouveront leur " résolution ». Revenons aux sacrifices d'holocauste, et citons (pour montrer tant le contraste que la continuité d'avec ces derniers) ce rituel de guérison taoïste attesté à la fin des Han : "Un prêtre se tenait aux côtés du malade en priant pour lui. Il transcrivait le nom de famille et le prénom de la personne souffrante ainsi que la liste des péchés qu'elle prenait sur son compte. Ce document était établi en trois exemplaires. Le premier était transmis au ciel, c'est-à-dire qu'on le plaçait au sommet d'une montagne. Le deuxième était enterré dans la terre et le troisième immergé dans l'eau " (cf. Seidel, ibid.). Le sacrifice devient holocauste textuel. Mais aussi, un nouveau rapport au texte fait de ce dernier un substitut du matériau sacrificiel.

Lus dans cette perspective, les textes classiques chinois pourraient révéler des anxiétés de nature plus proche de celles de l'Inde classique qu'on ne l'imaginerait au départ, anxiétés provoquées à la fois par l'exacerbation et la contestation $d u$ sacrifice sanglant. L'exaltation même du rituel et de sa portée cosmique pourrait traduire et la nécessité ressentie du sacrifice et la tentative de sortir de l'ordre qu'il clôt : partant du sacrifice, la construction ritualiste en précipite l'écroulement (The Broken World of Sacrifice, chapitre 2). Pour Heesterman, l'élaboration progressive du ritualisme résulte d'une conscience à la fois vive et comme dissimulée du caractère " agonistique » du sacrifice et du nécessaire dépassement de la « lutte à mort » qu'il exprime et perpétue ; le rituel fournit donc les règles $d u$ jeu qui désormais tiendra lieu d'affrontement entre mort et vie. C'est lorsqu'on a saisi que le développement du rite, lequel prend son origine dans le sacrifice, apprivoise ce dernier jusqu'à l'exténuer qu'on peut comprendre que le champ rituel est initialement, essentiellement champ de bataille. (À titre d'exemple, voir la courte analyse de l'espace rituel chinois que suggèrent à John Lagerwey la lecture du Zhouli et l'observation ethnographique - China. A Religious State, 2010, p. 7-10 et suiv. Les dispositions spatiales et mentales relevées par Lagerwey se retrouvent dans la lecture faite par Mark Edward Lewis des extraits pertinents du Zuozhuan: Sanctioned Violence in Early China, 1990, p. 16-17.) Xunzi, une fois encore, révèle mieux que bien d'autres l'anxiété qui travaille l'élaboration ritualiste : «Le rite, c'est ce qui est utilisé pour scrupuleusement (jin) administrer [zhi: gouverner, canaliser] [les matières concernant] la vie et la mort. » (Chapitre Lilun, par. 17)

\section{Conclusion}

Peut-on résumer le parcours accompli au cours de la lecture critique des ouvrages ici recensés ? Quelques points pour le moins ressortent assez clairement : 
- L'ouvrage de Gilles Boileau offre un panorama précieux des métamorphoses du Rite, de la Chine archaïque au début de la période impériale. Il montre comment la pratique rituelle (a) représente des degrés évolutifs de contrôle de l'espace naturel et d'appropriation des territoires; (b) structure (divise) et tente de rassembler un espace social dont la cohérence est sans cesse menacée, et pour ce faire institue des rôles, des codes, des procédures toujours renégociés ; (c) reflète et concourt à former un espace mental qui tend progressivement à faire du rituel même le lieu et l'opérateur des conjonctions entre microcosme et macrocosme ; et $(\mathrm{d})$ se révèle être elle-même un terrain de bataille sur lequel se jouent légitimation et appropriation du pouvoir.

- Pareille approche reste néanmoins à compléter par une prise en compte des différentes synthèses qui ont scandé la réflexion sur le rituel (les Analectes, le Xunzi, le Liji en premier lieu - la liste devrait être étendue). Ces synthèses ne nous fournissent pas seulement un matériau sur l'évolution des rituels et sur les enjeux vers lesquels cette évolution renvoyait. Ils offrent une réflexion autonome (riche, difficile, exigeant aujourd'hui une reprise herméneutique d'ampleur) autour du fondement symbolique du pouvoir, la nature, les conduites et la moralité humaines, le rapport entre l'éducation des sens et celle de l'intériorité, ou encore le mode de gestion des crises dans des communautés dont le degré de résilience reste toujours sujet à interrogation, entre autres questions abordées.

- L'étude de ces textes montre que le processus historique qui marque la période ne saurait être résumé en évoquant une quelconque «laïcisation » des rites, dont la fonction sociale opèrerait à terme de façon indépendante d'un substrat religieux ; les rituels équilibraient et intégraient les différents aspects de l'existence - sensorielle et festive, sociale, politique, religieuse, intérieure, morale, cosmique - en des synthèses créatives dont le ressort ultime était différemment interprété par les écrits et les courants dont il nous reste témoignage.

- Les rites se donnent à lire comme une rationalité opératoire. Ils restent potentiellement sujets à échec, car cette rationalité travaille sur l'impermanence des affaires humaines, l'inconnaissable du monde extra-humain, la fragilité du lien social. En même temps, les échecs participent du processus d'évolution des synthèses ritualistes.

- En leur principe, les rites sont sans doute inséparables d'un système mantique. L'évolution de ce dernier vers une rationalité divinatoire qui s'autonomise des occurrences rituelles dans lesquelles elle est mise en œuvre élargit parallèlement le champ d'action du rituel, sans que la relation organique entre rationalité divinatoire et rationalité opératoire (rituelle) soit jamais coupée.

- Cette rationalité intègre un travail sur l'affectivité (la musique) et, de manière corrélative et progressive, sur l'intériorisation des conduites. On pourrait même suggérer que, plus le rite se révèle sujet à échec dans l'effectuation de la cohésion sociale, plus l'accent se porte sur son efficace dans la correction 
et l'approfondissement des conduites. La relecture du rite ainsi entreprise par Confucius et ses premiers disciples va libérer l'espace pour de nouvelles synthèses mentales. Dans ces différentes synthèses, la dimension rituelle va prendre des formes très contrastées, mais en toutes elle restera un point nodal pour penser et représenter ensemble les articulations religieuses, politiques et morales du lien social.

- Il demeure pourtant dans ces textes comme la trace d'un secret du rite. J'ai suggéré que les élaborations incessantes autour de la question rituelle dont témoignent nos documents renvoyaient au fait suivant; si divination, sacrifice et rituel sont liés en leur principe, l'évolution rituelle va travailler à " exténuer " le caractère agonistique du sacrifice sans jamais y vraiment parvenir. Le rituel joue " avec " et " contre " le sacrifice : les constructions effectuées autour du $l i$ en termes spirituels et moraux (Confucius, Mencius), artificialistes (Xunzi) ou cosmologiques (Lüshi chunqiu, certains passages du Zhuangzi et du Hanfeizi) distancient toutes le rituel de ses origines sacrificielles sans pour autant l'instituer en substitut de ce dernier. Durant la période des Han postérieurs, la double révolution taoïste et bouddhiste changera pour partie les termes du débat, portant à maturation la proposition restée implicite dans la tradition ritualiste d'un rituel non sacrificiel. Néanmoins, la fusion entre cultes locaux et église taoïste, comme la reconstitution au début des Tang d'un "État-Église » fondé cette fois sur l'unité des «trois enseignements " (confucianisme, bouddhisme, taoïsme), vont réactiver la nature agonistique et sacrificielle des cultes liés à la gestion et à la protection du territoire étatique et de ceux des communautés locales (voir notamment Lagerwey, op. cit., p. 69-70, 173-174). J'ai suggéré ailleurs que le thème cannibale présent chez nombre d'auteurs chinois modernes et contemporains (Lu Xun, Mo Yan, A Cheng, Yu Hua, Yan Lianke) travaille à dévoiler la logique sacrificielle à l'œuvre dans une structure sociopolitique que lesdits sacrifices sacralisent (L'Empire sans milieu, 2010, p. 48-53). L'univers et les thèmes de ces œuvres évoquent parfois le matériau sur lequel Gilles Boileau reconstitue le monde social et rituel de la Chine antique.

Quoi qu'il en soit de la validité ultime du modèle ici suggéré, il permet du moins de circuler entre les études portant sur le rituel et le ritualisme chinois, d'une part, et celles qui concernent les mondes indien, gréco-romain ou sémitique, d'autre part. Notons en finale que l'idée selon laquelle le fondement du rituel reste étrangement occulté n'est peut-être pas absente de la tradition chinoise elle-même : "Interrogé sur le sens du grand Sacrifice à l'Ancêtre royal (di), Confucius répondit : "Je ne sais pas. Celui qui le saurait trouverait le gouvernement de l'Empire aussi simple que ceci.” Et il tourna sa paume. » (Analectes $3,11)$

Benoît VERMANDER Université Fudan (Shanghai), Faculté de Philosophie mdwei@fudan.edu.cn 
